\title{
Two-step Maruyama schemes for nonlinear stochastic differential delay equations
}

\author{
Dongxia Lei ${ }^{\mathrm{a}}$, Xiaofeng Zong ${ }^{\mathrm{b}}$, Junhao $\mathrm{Hu}^{\mathrm{c}, *}$ \\ a School of Mathematics and Statistics, Huazhong University of Science and Technology, Wuhan, 430074, China. \\ ${ }^{b}$ School of Automation, China University of Geosciences, Wuhan, 430074, China. \\ ${ }^{c}$ School of Mathematics and Statistics, South-Central University for Nationalities, Wuhan, 430074, China.
}

Communicated by X. Z. Liu

\begin{abstract}
This work concerns the two-step Maruyama schemes for nonlinear stochastic differential delay equations (SDDEs). We first examine the strong convergence rates of the split two-step Maruyama scheme and linear two-step Maruyama scheme (including Adams-Bashforth and Adams-Moulton schemes) for nonlinear SDDEs with highly nonlinear delay variables, then we investigate the exponential mean square stability and exponential decay rates of the two classes of two-step Maruyama schemes. These results are important for three reasons: first, the convergence rates are established under the non-global Lipschitz condition; second, these stability results show that these two-step Maruyama schemes can not only reproduce the exponential mean square stability, but also preserve the bound of Lyapunov exponent for sufficient small stepsize; third, they are also suitable for the corresponding two-step Maruyama methods of stochastic ordinary differential equations (SODEs). (C)2017 All rights reserved.
\end{abstract}

Keywords: Stochastic differential equations (SDEs), two-step Maruyama schemes, strong convergence rate, exponential mean square stability.

2010 MSC: $65 \mathrm{C} 30,93 \mathrm{E} 15,60 \mathrm{H} 35$.

\section{Introduction}

Most systems in science and industry are perturbed by some random environmental effects and the future state will often be related to past states except for the present states of the systems. Stochastic differential delay equations (SDDEs) have been used to model such systems and have the form

$$
d x(t)=f(x(t), x(t-\tau)) d t+g(x(t), x(t-\tau)) d w(t), \quad t>0
$$

with the initial data $x(t)=\varphi(t) \in C_{\widetilde{F}_{0}}^{b}\left([-\tau, 0] ; \mathbb{R}^{\mathfrak{n}}\right), t \in[-\tau, 0]$, where the delay $\tau>0$ is a fixed positive constant, the drift $f: \mathbb{R}^{n} \times \mathbb{R}^{n} \mapsto \mathbb{R}^{n}$ and the diffusion $g: \mathbb{R}^{n} \times \mathbb{R}^{n} \mapsto \mathbb{R}^{n \times d}$ are borel measurable, and $w(t)$ is a d-dimensional Brownian motion. When the delay vanishes, (1.1) is called stochastic ordinary

\footnotetext{
*Corresponding author

Email addresses: dongxialei@hust.edu.cn (Dongxia Lei), xfzong87816@gmail.com (Xiaofeng Zong), junhaohu74@163.com (Junhao $\mathrm{Hu}$ )

doi:10.22436/jnsa.010.10.11
}

Received 2017-08-23 
differential equations (SODEs). Indeed, SDDEs have come to play an important role in many branches of science and industry, see [1,3,10,14, 20, 26, 30] for example.

Due to that most of nonlinear stochastic differential equations (SDEs), including SODEs and SDDEs, do not have the explicit solutions, numerical solutions became an area of particular interest. So far, there is an extensive literature concerned with numerical analysis for SDEs and the key books in this area are $[18,22,23]$. In judging the quality of a numerical scheme, it is necessary to examine its convergence and stability. Abundant achievements have been made in the research of the strong convergence and exponential mean square stability of the one-step schemes. The linear mean square stability of thetaEuler methods and theta-Milstein schemes was investigated in $[4,11,19,24]$ and the strong convergence and exponential mean square stability of nonlinear SDEs with different Lipschitz conditions were well studied in [13, 15-17, 28, 29, 31-33].

Linear multi-step methods for SDEs also received a lot of attention. Buckwar and Winkler [6, 7] investigated the linear multi-step Maruyama methods for SODEs with small noise. Sickenberger [25] studied mean-square convergence of stochastic multi-step methods with variable step-size for SODEs. Buckwar and Winkler [8] investigated the mean square convergence of linear multi-step Maruyama methods for SDDEs under global Lipschitz condition. However, these convergence results are all in the sense of mean square and the strong convergence (or strong $\mathrm{L}^{p}$ convergence) has not be proved. Stability analysis is another research interest of the multi-step methods. By Lyapunov-type functionals, the asymptotic meansquare stability of the two-step Maruyama methods for linear SODEs was investigated in [5]. Necessary and sufficient conditions in terms of the parameters of the two-step Maruyama schemes guaranteeing their mean square stability were derived for linear SODEs in [27]. Exponential mean square stability of the two-step Maruyama schemes for linear SDDEs was studied in [9]. However, the mean square stability analysis of the multi-step schemes has not been established for nonlinear SODE and SDDEs.

The current article studies strong convergence rates and exponential mean square stability of the split two-step Maruyama and linear two-step Maruyama schemes for nonlinear SDDEs. The strong convergence rates are established for SDDEs with highly nonlinear delay variables. For the stability analysis, we address the following two questions:

(Q1) If the SDE is exponentially mean square stable, whether the two-step Maruyama methods can preserve this stability property.

(Q2) If the two-step Maruyama methods inherit the exponential mean square stability of the SDE, whether the exponential decay rate of exact solutions can be preserved as the stepsize $\Delta \rightarrow 0$.

The rest of the paper is organized as follows. Section 2 begins with notations and introduces the split two-step Maruyama and linear two-step Maruyama schemes for SDDEs. Section 3 gives the estimation of the moment and establishes the strong convergence rates of the two classes of two-step Maruyama schemes. Section 4 devotes to investigate the exponential mean square stability and the exponential decay rate of these two-step Maruyama schemes.

\section{Notations and preliminaries}

Throughout this paper, unless otherwise specified, we use the following notations. Let $|\cdot|$ denote both the Euclidean norm in $\mathbb{R}^{n}$ and the trace (or Frobenius) norm in $\mathbb{R}^{n \times d}$. If $A$ is a vector or matrix, its transpose is denoted by $A^{\top}$. If $A$ is a matrix, its trace norm is denoted by $|A|=\sqrt{\operatorname{trace}\left(A^{\top} A\right)}$. $a \vee b$ represents $\max \{a, b\}$ and $a \wedge b$ denotes $\min \{a, b\}$. Let $(\Omega, \mathfrak{F}, \mathbb{P})$ be a complete probability space with a filtration $\left\{\mathfrak{F}_{\mathrm{t}}\right\}_{\mathrm{t} \geqslant 0}$ satisfying the usual conditions, that is, it is right continuous and increasing while $\mathfrak{F}_{0}$ contains all $\mathbb{P}$-null sets. Let $w(t)$ be a d-dimensional Brownian motion defined on this probability space.

The classic linear two-step Maruyama schemes for SDDE (1.1) was introduced in [8] and have the form

$$
\begin{aligned}
y_{k+1}+\alpha_{1} y_{k}+\alpha_{0} y_{k-1}= & \Delta\left(\beta_{2} f\left(y_{k+1}, y_{k+1-m}\right)+\beta_{1} f\left(y_{k}, y_{k-m}\right)+\beta_{0} f\left(y_{k-1}, y_{k-1-m}\right)\right) \\
& +r_{1} g\left(y_{k}, y_{k-m}\right) \Delta w_{k}+r_{0} g\left(y_{k-1}, y_{k-1-m}\right) \Delta w_{k-1}
\end{aligned}
$$


with $y_{k}=\varphi(k \Delta)$ for $k=-m, \ldots, 0$, where $\Delta=\tau / m$ for a positive integer $m$ is the constant stepsize, $\Delta w_{k}:=w((k+1) \Delta)-w(k \Delta)$ is Brownian increment, and $\alpha_{1}, \alpha_{0}, \beta_{2}, \beta_{1}, \beta_{0}, r_{1}, r_{0}$ are real parameters; notice that the coefficient $\alpha_{2}=1$ of $y_{k+1}$ has been normalized. In order to ensure the convergence and consistency, the following conditions will be needed: (1) Ahlquists root condition, i.e., the polynomial $\rho(r)=r^{2}+\alpha_{1} r+\alpha_{0}$ has all its zeros in the unit disk $|r| \leqslant 1$ without multiple zeros on the border $|r|=0$. (2) Deterministic consistency conditions, i.e., $1+\alpha_{1}+\alpha_{0}=0,2+\alpha_{1}=\beta_{0}+\beta_{1}+\beta_{2}$. (3) Stochastic consistency conditions, i.e., $r_{1}=1, r_{0}=1+\alpha_{1}$.

In this paper, we consider the case: $\alpha_{0}=0, \alpha_{1}=-1$, then the two-step Maruyama method (2.1) can be rewriten as

$$
y_{k+1}=y_{k}+\Delta\left[\beta_{2} f\left(y_{k+1}, y_{k+1-m}\right)+\beta_{1} f\left(y_{k}, y_{k-m}\right)+\beta_{0} f\left(y_{k-1}, y_{k-1-m}\right)\right]+g\left(y_{k}, y_{k-m}\right) \Delta w_{k} .
$$

When $\beta_{2}=0, \beta_{1}=\frac{3}{2}, \beta_{0}=-\frac{1}{2}$, (2.2) is Adams-Bashforth Maruyama scheme, and when $\beta_{2}=\frac{5}{12}, \beta_{1}=$ $\frac{8}{12}, \beta_{0}=-\frac{1}{12}$, (2.2) is Adams-Moulton Maruyama scheme.

In order to examine the strong convergence and stability of (2.2), we introduce the split two-step Maruyama (STSM) scheme $z_{k}$, defined by

$$
\left\{\begin{array}{l}
y_{k}=z_{k}+\beta_{2} f\left(y_{k}, y_{k-m}\right) \Delta-\beta_{0} f\left(y_{k-1}, y_{k-1-m}\right) \Delta \\
z_{k+1}=z_{k}+f\left(y_{k}, y_{k-m}\right) \Delta+g\left(y_{k}, y_{k-m}\right) \Delta w_{k}, \quad k=0,1,2, \ldots
\end{array}\right.
$$

with $z_{0}=y_{0}-\beta_{2} f\left(y_{0}, y_{-m}\right) \Delta+\beta_{0} f\left(y_{-1}, y_{-m-1}\right) \Delta, y_{-m-1}=y_{-m}, \Delta w_{k}:=w((k+1) \Delta)-w(k \Delta)$ is Brownian increment. It is easy to see that approximation $y_{k}$ in (2.3) is actually the linear two-step Maruyama (LTSM) scheme (2.2) with $\beta_{1}=1-\beta_{2}-\beta_{0}$.

Note that when $\beta_{0}=0, \beta_{2}, \beta_{1} \geqslant 0,(2.2)$ and (2.3) are the stochastic linear theta-Euler and the split-step theta-Euler schemes, respectively, which were well investigated for SDDEs in [33]. In order to distinguish, we call (2.2) linear two-step Maruyama (LTSM) scheme.

Noting that the two classes of two-step schemes (2.3) and (2.2) are semi-implicit when $\beta_{2} \neq 0$, to guarantee that they are well-defined, we need the one-sided Lipschitz condition

$$
\langle x-y, f(x, z)-f(y, z)\rangle \leqslant \mu|x-y|^{2}
$$

for any $x, y, z \in \mathbb{R}^{n}$, and restrict the stepsize $\Delta$ satisfying $\left|\beta_{2}\right| \mu \Delta<1$, which implies that the implicit equation

$$
y=c+\theta \Delta f(y, \bar{y})
$$

admits a unique solution $y=F_{\beta_{2}, \theta}(c, \bar{y})$ for any fixed $c, \bar{y} \in \mathbb{R}^{n}$ (see [12]).

Buckwar and Winkler [8] showed that the LTSM scheme (2.2) is mean square convergent under the global Lipschitz condition on coefficients $f$ and $g$. Then it is easy to see from $z_{k}=y_{k}-\beta_{2} f\left(y_{k}, y_{k-m}\right) \Delta+$ $\beta_{0} f\left(y_{k-1}, y_{k-1-m}\right)$ that STSM scheme is also mean square convergent under the same conditions. In the following, we can prove that LTSM and STSM schemes is strongly convergent to the exact solution with order 0.5 under the non-global Lipschitz condition on the delay term.

\section{Moment boundedness and strong convergence rate}

In this section, let

$$
0 \leqslant V_{i}(x, y) \leqslant L_{i}\left(1+|x|^{q_{i}}+|y|^{q_{i}}\right), i=1,2,
$$

and assume that $f$ and $g$ hold the following assumption.

Assumption 3.1. Assume $f(x, y), g(x, y)$ are $C^{1}$ functions and there exist constants $a, b>0$ such that

$$
\begin{aligned}
& \left|f\left(x_{1}, y_{1}\right)-f\left(x_{2}, y_{2}\right)\right| \leqslant a\left|x_{1}-x_{2}\right|+v_{1}\left(y_{1}, y_{2}\right)\left|y_{1}-y_{2}\right| \\
& \left|g\left(x_{1}, y_{1}\right)-g\left(x_{2}, y_{2}\right)\right| \leqslant b\left|x_{1}-x_{2}\right|+v_{2}\left(y_{1}, y_{2}\right)\left|y_{1}-y_{2}\right|
\end{aligned}
$$

for all $x, y, x_{1}, y_{1}, x_{2}, y_{2} \in \mathbb{R}^{n}$. 
From Assumption 3.1, we have the following estimates

$$
\begin{aligned}
\langle x, f(x, y)\rangle & =\langle x-0, f(x, y)-f(0, y)\rangle+\langle x, f(0, y)-f(0,0)\rangle+\langle x, f(0,0)\rangle \\
& \leqslant(1+a)|x|^{2}+V_{1}(y, 0)^{2}|y|^{2}+\frac{1}{2}|f(0,0)|^{2}, \\
|f(x, y)|^{2} & \leqslant 2|f(x, y)-f(0,0)|^{2}+2|f(0,0)|^{2} \leqslant 2 a^{2}|x|^{2}+2 V_{1}(y, 0)^{2}|y|^{2}+2|f(0,0)|^{2},
\end{aligned}
$$

and

$$
|g(x, y)|^{2} \leqslant 2|g(x, y)-g(0,0)|^{2}+2|g(0,0)|^{2} \leqslant 2 b^{2}|x|^{2}+2 V_{2}(y, 0)^{2}|y|^{2}+2|g(0,0)|^{2} .
$$

For simplicity, we let $K=\max \left\{2+2 a, 2 a^{2},|f(0,0)|^{2}, 2 b^{2}, 2|g(0,0)|^{2}\right\}$ and $V(y)=\sqrt{2} \max \left\{V_{1}(y, 0), V_{2}(y, 0)\right\}$, then

$$
2\langle x, f(x, y)\rangle \vee|f(x, y)|^{2} \vee|g(x, y)|^{2} \leqslant K\left(1+|x|^{2}\right)+V(y)^{2}|y|^{2} .
$$

The following theorem shows that under Assumption 3.1, Eq. (1.1) has a global solution (see [2]).

Theorem 3.2. If Assumption 3.1 holds, then there exists a unique global solution for Eq. (1.1). Moreover, for all $\mathrm{p} \geqslant 2, \mathrm{~T}>0$, the solution holds the following property

$$
\mathbb{E}\left[\sup _{t \in[0, T]}|x(t)|^{p}\right] \leqslant C(\varphi, p, T),
$$

where $\mathrm{C}(\varphi, \mathrm{p}, \mathrm{T})$ is a positive constant depending on the initial data $\varphi$ and $\mathrm{p}, \mathrm{T}$.

Note that condition (3.2) implies (2.4) with $\mu=b$. We define $\bar{K}=\left|\beta_{2}\right|+\left|\beta_{0}\right|+K\left|\beta_{2}\right|$, then for $\Delta<\Delta^{*}=$ $1 / \bar{K}$, the two-step schemes (2.3) and (2.2) are all well-defined. In order to obtain the convergence rate, we firstly examine the moment boundedness of the two-step schemes.

Theorem 3.3. Let Assumption 3.1 hold and $\Delta<\Delta^{*}$. Then for each integer $p \geqslant 2$,

$$
\mathbb{E}\left[\sup _{k \Delta \in[0, T]}\left|z_{k}\right|^{p}\right] \leqslant C \text { and } \mathbb{E}\left[\sup _{k \Delta \in[0, T]}\left|y_{k}\right|^{p}\right] \leqslant C .
$$

Proof. By STSM scheme (2.3), we have

$$
\begin{aligned}
\left|z_{k+1}\right|^{2}= & \left|z_{k}\right|^{2}+\left|f\left(y_{k}, y_{k-m}\right)\right|^{2} \Delta^{2}+\Delta w_{k}^{\top} g^{\top}\left(y_{k}, y_{k-m}\right) g\left(y_{k}, y_{k-m}\right) \Delta w_{k} \\
& +2 \Delta z_{k}^{\top} f\left(y_{k}, y_{k-m}\right)+2\left(z_{k}+f\left(y_{k}, y_{k-m}\right) \Delta\right)^{\top} g\left(y_{k}, y_{k-m}\right) \Delta w_{k} .
\end{aligned}
$$

Substituting $z_{k}=y_{k}-\beta_{2} f\left(y_{k}, y_{k-m}\right) \Delta+\beta_{0} f\left(y_{k-1}, y_{k-1-m}\right) \Delta$ into the last two terms in (3.6) yields

$$
\begin{aligned}
\left|z_{k+1}\right|^{2}= & \left|z_{k}\right|^{2}+2 \Delta y_{k}^{\top} f\left(y_{k}, y_{k-m}\right)+\left(1-2 \beta_{2}\right)\left|f\left(y_{k}, y_{k-m}\right)\right|^{2} \Delta^{2} \\
& -2 \beta_{0} f\left(y_{k-1}, y_{k-1-m}\right)^{\top} f\left(y_{k}, y_{k-m}\right) \Delta^{2}+\left|g\left(y_{k}, y_{k-m}\right) \Delta w_{k}\right|^{2} \\
& +2\left[y_{k}+\left(1-\beta_{2}\right) f\left(y_{k}, y_{k-m}\right) \Delta-\beta_{0} f\left(y_{k-1}, y_{k-1-m}\right) \Delta\right]^{\top} g\left(y_{k}, y_{k-m}\right) \Delta w_{k},
\end{aligned}
$$

which implies

$$
\begin{aligned}
\left|z_{k+1}\right|^{2} \leqslant & \left|z_{0}\right|^{2}+2 \Delta \sum_{i=0}^{k}\left[y_{i}^{\top} f\left(y_{i}, y_{i-m}\right)\right]+\left(1-2 \beta_{2}+\left|\beta_{0}\right|\right) \Delta^{2} \sum_{i=0}^{k}\left|f\left(y_{i}, y_{i-m}\right)\right|^{2} \\
& +\left|\beta_{0}\right| \Delta^{2} \sum_{i=0}^{k}\left|f\left(y_{i-1}, y_{i-1-m}\right)\right|^{2}+\sum_{i=0}^{k}\left|g\left(y_{i}, y_{i-m}\right) \Delta w_{i}\right|^{2}+2 \sum_{i=0}^{k} y_{i}^{\top} g\left(y_{i}, y_{i-m}\right) \Delta w_{k} \\
& +2\left(1-\beta_{2}\right) \Delta \sum_{i=0}^{k} f\left(y_{i}, y_{i-m}\right)^{\top} g\left(y_{i}, y_{i-m}\right) \Delta w_{i} \\
& -2 \beta_{0} \Delta \sum_{i=0}^{k} f\left(y_{i-1}, y_{i-1-m}\right)^{\top} g\left(y_{i}, y_{i-m}\right) \Delta w_{i} .
\end{aligned}
$$


Resorting to inequalities (3.5), we have from (3.7) that

$$
\begin{aligned}
\left|z_{k+1}\right|^{2} \leqslant & C+C \Delta \sum_{i=0}^{k}\left|y_{i}\right|^{2}+C \Delta \sum_{i=0}^{k} V\left(y_{i-m}\right)^{2}\left|y_{i-m}\right|^{2} \\
& +\sum_{i=0}^{k}\left|g\left(y_{i}, y_{i-m}\right) \Delta w_{i}\right|^{2}+2 \sum_{i=0}^{k} y_{i}^{\top} g\left(y_{i}, y_{i-m}\right) \Delta w_{k} \\
& +2\left(1-\beta_{2}\right) \Delta \sum_{i=0}^{k} f\left(y_{i}, y_{i-m}\right)^{\top} g\left(y_{i}, y_{i-m}\right) \Delta w_{i} \\
& -2 \beta_{0} \Delta \sum_{i=0}^{k} f\left(y_{i-1}, y_{i-1-m}\right)^{\top} g\left(y_{i}, y_{i-m}\right) \Delta w_{i} .
\end{aligned}
$$

Raising both sides to the power $p \geqslant 1$, we have

$$
\begin{aligned}
\frac{1}{8^{p-1}}\left|z_{k+1}\right|^{2 p} \leqslant & C+C \Delta^{p}\left(\sum_{i=0}^{k}\left|y_{i}\right|^{2}\right)^{p}+C \Delta^{p}\left(\sum_{i=0}^{k} v\left(y_{i-m}\right)^{2}\left|y_{i-m}\right|^{2}\right)^{p} \\
& +\left(\sum_{i=0}^{k}\left|g\left(y_{i}, y_{i-m}\right) \Delta w_{i}\right|^{2}\right)^{p}+2^{p}\left|\sum_{i=0}^{k} y_{i}^{\top} g\left(y_{i}, y_{i-m}\right) \Delta w_{k}\right|^{p} \\
& +C\left|\sum_{i=0}^{k} f\left(y_{i}, y_{i-m}\right)^{\top} g\left(y_{i}, y_{i-m}\right) \Delta w_{i}\right|^{p} \\
& +C\left|\sum_{i=0}^{k} f\left(y_{i-1}, y_{i-1-m}\right)^{\top} g\left(y_{i}, y_{i-m}\right) \Delta w_{i}\right|^{p} .
\end{aligned}
$$

It is easy to see that for any $0<\mathrm{l}<\mathrm{N}$

$$
\mathbb{E}\left[\sup _{0 \leqslant k \leqslant l}\left(\sum_{i=0}^{k}\left|y_{i}\right|^{2}\right)^{p}\right] \leqslant N^{p-1} \mathbb{E}\left[\sum_{i=0}^{l}\left|y_{i}\right|^{2 p}\right]
$$

and

$$
\begin{aligned}
\mathbb{E}\left[\sup _{0 \leqslant k \leqslant l}\left(\sum_{i=0}^{k} \mathrm{~V}\left(y_{i-m}\right)^{2}\left|y_{i-m}\right|^{2}\right)^{p}\right] & \leqslant N^{p-1} \mathbb{E}\left[\sum_{i=0}^{l} V\left(y_{i-m}\right)^{2 p}\left|y_{i-m}\right|^{2 p}\right] \\
& \leqslant C N^{p-1}\left(\mathbb{E}\left|y_{i-m}\right|^{2 p}+\mathbb{E}\left|y_{i-m}\right|^{2 p\left(1+q_{1}\right)}+\mathbb{E}\left|y_{i-m}\right|^{2 p\left(1+q_{2}\right)}\right) .
\end{aligned}
$$

Note that $\mathfrak{y}_{i}, y_{i-m}$ are $\mathfrak{F}_{i \Delta}$-measurable while $\Delta w_{\mathfrak{i}}$ is $\mathfrak{F}_{i \Delta}$ independent. Using (3.4), we have

$$
\begin{aligned}
\mathbb{E}\left(\sup _{0 \leqslant k \leqslant l} \sum_{i=0}^{k}\left|g\left(y_{i}, y_{i-m}\right)\right|^{2}\left|\Delta w_{i}\right|^{2}\right)^{p} & \leqslant N^{p-1} \mathbb{E}\left[\sup _{0 \leqslant k \leqslant l} \sum_{i=0}^{k}\left|g\left(y_{i}, y_{i-m}\right) \Delta w_{i}\right|^{2 p}\right] \\
& =N^{p-1} \mathbb{E}\left[\sum_{i=0}^{l}\left|g\left(y_{i}, y_{i-m}\right) \Delta w_{i}\right|^{2 p}\right] \\
& =N^{p-1} \sum_{i=0}^{l} \mathbb{E}\left|g\left(y_{i}, y_{i-m}\right)\right|^{2 p} \mathbb{E}\left|\Delta w_{i}\right|^{2 p} \\
& \leqslant C \Delta \sum_{i=0}^{l} \mathbb{E}\left[K\left(1+\left|y_{i}\right|^{2}\right)+V_{2}\left(y_{i-m}, 0\right)^{2}\left|y_{i-m}\right|^{2}\right]^{p}
\end{aligned}
$$




$$
\leqslant \mathrm{C}+\mathrm{C} \Delta \sum_{i=0}^{\mathrm{l}} \mathbb{E}\left[\left|\mathrm{y}_{i}\right|^{2 \mathrm{p}}\right]+\mathrm{C} \Delta \sum_{i=0}^{\mathrm{l}}\left(\mathbb{E}\left|\mathrm{y}_{i-\mathrm{m}}\right|^{2 \mathrm{p}}+\mathbb{E}\left|\mathrm{y}_{i-\mathrm{m}}\right|^{2 \mathrm{p}\left(1+\mathrm{q}_{2}\right)}\right)
$$

The Burkholder-Davis-Gundy inequality gives

$$
\begin{aligned}
& \mathbb{E}\left[\sup _{0 \leqslant k \leqslant l}\left|\sum_{i=0}^{k} y_{i}^{\top} g\left(y_{i}, y_{i-m}\right) \Delta w_{i}\right|^{p}\right] \\
& \leqslant C \mathbb{E}\left[\sum_{i=0}^{l}\left|y_{i}\right|^{2}\left|g\left(y_{i}, y_{i-m}\right)\right|^{2} \Delta\right]^{p / 2} \\
& \leqslant C \Delta^{p / 2} m^{p / 2-1} \mathbb{E} \sum_{i=0}^{l}\left|y_{i}\right|^{p}\left[1+\left|y_{i}\right|^{2}+v_{2}\left(y_{i-m}, 0\right)^{2}\left|y_{i-m}\right|^{2}\right]^{p / 2} \\
& \leqslant C+C \Delta \sum_{i=0}^{l} \mathbb{E}\left[\left|y_{i}\right|^{2 p}\right]+C \Delta \sum_{i=0}^{l}\left(\mathbb{E}\left|y_{i-m}\right|^{2 p}+\mathbb{E}\left|y_{i-m}\right|^{2 p\left(1+q_{2}\right)}\right], \\
& \mathbb{E}\left[\sup _{0 \leqslant k \leqslant l}\left|\sum_{i=0}^{k} f\left(y_{i}, y_{i-m}\right)^{\top} g\left(y_{i}, y_{i-m}\right) \Delta w_{i}\right|^{p}\right] \\
& \leqslant C \mathbb{E}\left[\sum_{i=0}^{l}\left|f\left(y_{i}, y_{i-m}\right)\right|^{2}\left|g\left(y_{i}, y_{i-m}\right)\right|^{2} \Delta\right]^{p / 2} \\
& \leqslant C \Delta^{p / 2} l^{p / 2-1} \mathbb{E} \sum_{i=0}^{l}\left(\left|f\left(y_{i}, y_{i-m}\right)\right|^{2 p}+\left|g\left(y_{i}, y_{i-m}\right)\right|^{2 p}\right) \\
& \leqslant C+C \Delta \sum_{i=0}^{l}\left(\mathbb{E}\left|y_{i}\right|^{2 p}+\mathbb{E}\left|y_{i-m}\right|^{2 p}+\mathbb{E}\left|y_{i-m}\right|^{2 p\left(1+q_{1}\right)}+\mathbb{E}\left|y_{i-m}\right|^{2 p\left(1+q_{2}\right)}\right),
\end{aligned}
$$

and similarly

$$
\begin{aligned}
& \mathbb{E}\left[\sup _{0 \leqslant k \leqslant l}\left|\sum_{i=0}^{k} f\left(y_{i-1}, y_{i-1-m}\right)^{\top} g\left(y_{i}, y_{i-m}\right) \Delta w_{i}\right|^{p}\right] \\
& \leqslant C+C \Delta \sum_{i=0}^{l}\left(\mathbb{E}\left|y_{i}\right|^{2 p}+\mathbb{E}\left|y_{i-m}\right|^{2 p}+\mathbb{E}\left|y_{i-m}\right|^{2 p\left(1+q_{1}\right)}+\mathbb{E}\left|y_{i-m}\right|^{2 p\left(1+q_{2}\right)}\right),
\end{aligned}
$$

where we also used (3.5) and the Hölder inequality. Combining (3.8)-(3.13) yields

$$
\begin{aligned}
\mathbb{E}\left[\sup _{0 \leqslant k \leqslant l+1}\left|z_{k}\right|^{2 p}\right] \leqslant & C+C \Delta \sum_{i=0}^{l} \mathbb{E}\left[\left|y_{i}\right|^{2 p}\right]+C \Delta \sum_{i=0}^{l} \mathbb{E}\left[\left|y_{i-m}\right|^{2 p}\right] \\
& +C \Delta \sum_{i=0}^{l} \mathbb{E}\left[\left|y_{i-m}\right|^{2 \mathfrak{p}\left(1+q_{1}\right)}\right]+C \Delta \sum_{i=0}^{l} \mathbb{E}\left[\left|y_{i-m}\right|^{2 p\left(1+q_{2}\right)}\right] .
\end{aligned}
$$

Then, using $z_{k}=y_{k}-\beta_{2} f\left(y_{k}, y_{k-m}\right) \Delta+\beta_{0} f\left(y_{k-1}, y_{k-1-m}\right) \Delta$ and (3.3), again, we deduce that for $\theta \Delta<$ $1 / K$,

$$
\left|z_{k}\right|^{2}=\left|y_{k}\right|^{2}-2 y_{k}^{\top}\left[\beta_{2} f\left(y_{k}, y_{k-m}\right) \Delta+\beta_{0} f\left(y_{k-1}, y_{k-1-m}\right) \Delta\right]
$$




$$
\begin{aligned}
& +\left|\beta_{2} f\left(y_{k}, y_{k-m}\right) \Delta+\beta_{0} f\left(y_{k-1}, y_{k-1-m}\right) \Delta\right|^{2} \\
\geqslant & \left|y_{k}\right|^{2}-2 y_{k}^{\top}\left[\beta_{2} f\left(y_{k}, y_{k-m}\right) \Delta+\beta_{0} f\left(y_{k-1}, y_{k-1-m}\right) \Delta\right],
\end{aligned}
$$

that is,

$$
\begin{aligned}
\left|y_{k}\right|^{2} \leqslant & \left|z_{k}\right|^{2}+2 y_{k}^{\top}\left[\beta_{2} f\left(y_{k}, y_{k-m}\right) \Delta+\beta_{0} f\left(y_{k-1}, y_{k-1-m}\right) \Delta\right] \\
\leqslant & \left|z_{k}\right|^{2}+\left|\beta_{2}\right| \Delta\left(\left|y_{k}\right|^{2}+\left|f\left(y_{k}, y_{k-m}\right)\right|^{2}\right)+\left|\beta_{0}\right| \Delta\left(\left|y_{k}\right|^{2}+\left|f\left(y_{k-1}, y_{k-1-m}\right)\right|^{2}\right) \\
\leqslant & \left|z_{k}\right|^{2}+K \Delta+\left(\left|\beta_{2}\right|+\left|\beta_{0}\right|+K\left|\beta_{2}\right|\right) \Delta\left|y_{k}\right|^{2}+\left|\beta_{0}\right| \Delta\left|y_{k-1}\right|^{2} \\
& +2\left|\beta_{0}\right| \Delta V_{1}\left(y_{k-1-m}, 0\right)^{2}\left|y_{k-1-m}\right|^{2}+2\left|\beta_{2}\right| \Delta V_{1}\left(y_{k-m}, 0\right)^{2}\left|y_{k-m}\right|^{2}
\end{aligned}
$$

which implies

$$
\begin{aligned}
(1-\overline{\mathrm{K}} \Delta)\left|y_{k}\right|^{2} & =\left(1-\left(\left|\beta_{2}\right|+\left|\beta_{0}\right|+K\left|\beta_{2}\right|\right) \Delta\right)\left|y_{k}\right|^{2} \\
& \leqslant\left|z_{k}\right|^{2}+K \Delta+\left|\beta_{0}\right| \Delta\left|y_{k-1}\right|^{2}+2\left|\beta_{0}\right| \Delta V_{1}\left(y_{k-1-m}, 0\right)^{2}\left|y_{k-1-m}\right|^{2}+2\left|\beta_{2}\right| \Delta V_{1}\left(y_{k-m}, 0\right)^{2}\left|y_{k-m}\right|^{2}
\end{aligned}
$$

Using (3.1) and Assumption 3.1, we can obtain that for $\Delta<\Delta^{*}=1 / \overline{\mathrm{K}}$,

$$
\begin{aligned}
& (1-\overline{\mathrm{K}} \Delta)^{\mathrm{p}}\left|\mathrm{y}_{\mathrm{k}}\right|^{2 \mathrm{p}} \leqslant 5^{\mathrm{p}-1}\left(\left|z_{\mathrm{k}}\right|^{2 \mathrm{p}}+(\mathrm{KT})^{\mathrm{P}}+\left(2\left|\beta_{0}\right| \Delta\right)^{\mathrm{p}} \mathrm{L}_{1}^{2 \mathrm{p}}\left(1+\left|\mathrm{y}_{\mathrm{k}-1-\mathrm{m}}\right|^{\mathfrak{q}_{1}}\right)^{2 \mathrm{p}}\left|\mathrm{y}_{\mathrm{k}-1-\mathrm{m}}\right|^{2 \mathrm{p}}\right. \\
& \left.+\left|\beta_{0}\right|^{p} \Delta^{p}\left|y_{k-1}\right|^{2 p}+\left(2\left|\beta_{0}\right| \Delta\right)^{p} L_{1}^{2 p}\left(1+\left|y_{k-m}\right|^{q_{1}}\right)^{2 p}\left|y_{k-m}\right|^{2 p}\right) \\
& \leqslant 5^{p-1}\left|z_{k}\right|^{2 p}+C+C \Delta^{p}\left|y_{k-1-m}\right|^{2 p}+C \Delta^{p}\left|y_{k-1-m}\right|^{2 p\left(1+q_{1}\right)} \\
& +C \Delta^{\mathfrak{p}}\left|y_{k-1}\right|^{2 \mathfrak{p}}+C \Delta^{\mathfrak{p}}\left|y_{k-m}\right|^{2 p}+C \Delta^{p}\left|y_{k-m}\right|^{2 p\left(1+q_{1}\right)} \text {. }
\end{aligned}
$$

Combining (3.14) and (3.15) produces that for any $p \geqslant 1$

$$
\begin{aligned}
& (1-\overline{\mathrm{K}} \theta \Delta)^{\mathrm{p}} \mathbb{E}\left[\sup _{0 \leqslant k \leqslant l+1}\left|y_{k}\right|^{2 p}\right] \\
& \leqslant \mathrm{C}+\mathrm{C} \Delta \sum_{i=0}^{\mathrm{l}} \mathbb{E}\left|\mathrm{y}_{\mathrm{i}}\right|^{2 \mathrm{p}}+\mathrm{C} \Delta \sum_{i=0}^{\mathrm{l}} \mathbb{E}\left[\left|\mathrm{y}_{\mathrm{i}-\mathrm{m}}\right|^{2 \mathrm{p}}\right] \\
& +\mathrm{C} \Delta \sum_{i=0}^{\mathrm{l}} \mathbb{E}\left[\left|\mathrm{y}_{i-\mathrm{m}}\right|^{2 \mathrm{p}\left(1+\mathrm{q}_{1}\right)}\right]+\mathrm{C} \Delta \sum_{i=0}^{\mathrm{l}} \mathbb{E}\left[\left|\mathrm{y}_{i-\mathrm{m}}\right|^{2 \mathfrak{p}\left(1+\mathrm{q}_{2}\right)}\right] \\
& \leqslant \mathrm{C}+\mathrm{C} \Delta \sum_{i=0}^{\mathrm{l}} \mathbb{E}\left[\sup _{0 \leqslant k \leqslant i}\left|y_{k}\right|^{2 p}\right]+\mathrm{C} \Delta \sum_{i=0}^{\mathrm{l}} \mathbb{E}\left[\left|y_{i-m}\right|^{2 p\left(1+\mathrm{q}_{1}\right)}\right]+\mathrm{C} \Delta \sum_{i=0}^{\mathrm{l}} \mathbb{E}\left[\left|y_{i-m}\right|^{2 \mathfrak{p}\left(1+\mathrm{q}_{2}\right)}\right],
\end{aligned}
$$

where we also used the following inequality

$$
\sum_{i=0}^{l} \mathbb{E}\left[\left|y_{i-m}\right|^{2 p}\right]=\sum_{i=-m}^{-1} \mathbb{E}\left[\left|y_{i}\right|^{2 p}\right]+\sum_{i=0}^{l-m} \mathbb{E}\left[\left|y_{i}\right|^{2 p}\right] \leqslant \sum_{i=-m}^{-1} \mathbb{E}\left[\left|y_{i}\right|^{2 p}\right]+\sum_{i=0}^{l} \mathbb{E}\left[\left|y_{i}\right|^{2 p}\right] .
$$

Hence, by the discrete Gronwall inequality, we have that for any $p \geqslant 2$ and $l \leqslant N$,

$$
\mathbb{E}\left[\sup _{0 \leqslant k \leqslant l}\left|y_{k}\right|^{p}\right] \leqslant C+C \Delta\left\{\sum_{i=0}^{l-1} \mathbb{E}\left[\left|y_{i-m}\right|^{p\left(1+q_{1}\right)}\right]+\sum_{i=0}^{l-1} \mathbb{E}\left[\left|y_{i-m}\right|^{p\left(1+q_{2}\right)}\right]\right\} .
$$

Let $r_{1}=1+q_{1}, r_{2}=1+q_{2}$, and $\bar{r}=r_{1} \vee r_{2}(>1)$. For any fixed $p \geqslant 2$, we define

$$
p_{i}=([N / m]+2-i) p \bar{r}^{[N / m]+1-i}, i=1,2, \ldots,[N / m]+1,
$$


where $[a]$ denotes the integer part of the real number $a$. It is easy to see that $p_{i} \geqslant 2$,

$$
\overline{\mathrm{r}} \mathrm{p}_{\mathrm{i}+1}<\mathrm{p}_{\mathrm{i}} \text { and } \mathrm{p}_{[\mathrm{N} / \mathrm{m}]+1}=\mathrm{p}, i=1,2, \ldots,[\mathrm{N} / \mathrm{m}] \text {. }
$$

By (3.16) and $\varphi(t) \in \mathrm{C}_{\widetilde{F}_{0}}^{\mathrm{b}}\left([-\tau, 0] ; \mathbb{R}^{\mathfrak{n}}\right)$, we can easily obtain

$$
\mathbb{E}\left[\sup _{0 \leqslant k \leqslant m}\left|y_{k}\right|^{p_{1}}\right] \leqslant C,
$$

which together with (3.16) and Hölder's inequality yields

$$
\begin{aligned}
\mathbb{E}\left[\sup _{0 \leqslant k \leqslant 2 m}\left|y_{k}\right|^{p_{2}}\right] & \leqslant C+C \Delta\left\{\sum_{i=0}^{2 m-1} \mathbb{E}\left[\left|y_{i-m}\right|^{p_{2} r_{1}}\right]+\sum_{i=0}^{2 m-1} \mathbb{E}\left[\left|y_{i-m}\right|^{p_{2} r_{2}}\right]\right\} \\
& \leqslant C+C \Delta\left\{\sum_{i=0}^{2 m-1}\left(\mathbb{E}\left[\left|y_{i-m}\right|^{p_{1}}\right]\right)^{\frac{p_{2} r_{1}}{p_{1}}}+\sum_{i=0}^{2 m-1}\left(\mathbb{E}\left[\left|y_{i-m}\right|^{p_{1}}\right]\right)^{\frac{p_{2} r_{2}}{p_{1}}}\right\} \leqslant C .
\end{aligned}
$$

Since $N / m=T / \tau$ is finite, then repeating the previous procedures gives that for any $p \geqslant 2$

$$
\mathbb{E}\left[\sup _{0 \leqslant k \leqslant N}\left|y_{k}\right|^{p}\right] \leqslant \mathbb{E}\left[\sup _{0 \leqslant k \leqslant([N / m]+1) m}\left|y_{k}\right|^{p_{[N / m]+1}}\right] \leqslant C,
$$

which together with (3.14) implies

$$
\mathbb{E}\left[\sup _{0 \leqslant k \leqslant N}\left|z_{k}\right|^{p}\right] \leqslant C
$$

After the moment boundedness of the two-step Maruyama schemes, we now turn to their convergence analysis. Firstly, we need to establish the time continuous interpolations of the time discrete numerical approximation $\left\{z_{k}\right\}_{k} \geqslant 0$. For $t \in\left[t_{k}, t_{k+1}\right), k=0,1, \ldots, N-1$, we define the continuous approximate solutions $z(t)$ as follows

$$
z(t)=z\left(t_{k}\right)+\left(t-t_{k}\right) f\left(y_{k}, y_{k-m}\right)+g\left(y_{k}, y_{k-m}\right)\left(w(t)-w\left(t_{k}\right)\right),
$$

where $z(0)=z_{0}=y_{0}+\beta_{2} \Delta f\left(y_{0}, y(-\tau)\right)-\beta_{0} \Delta f\left(y_{-1}, y_{-m}\right), t_{k}=k \Delta$. For $t \in[-\tau, 0]$, we define $y(t)=$ $x(t)=\varphi(t)$. It is obvious that the continuous approximations $z(t)$ and $y(t)$ are $\mathfrak{F}_{t}$-measurable, moreover, $z\left(t_{k}\right)=z_{k}, y\left(t_{k}\right)=y_{k}$. In the following, we also use the following equivalent form of $z(t)$

$$
z(t)=z(0)+\int_{0}^{t} f(y(\check{s}), y(\check{s}-\tau)) d s+\int_{0}^{t} g(y(\check{s}), y(\check{s}-\tau)) d w(s),
$$

where $\breve{s}=t_{k}$ for $s \in\left[t_{k}, t_{k+1}\right)$. We call $z(t)$ the continuous extension of the discrete processes $z_{k}$.

Firstly, we investigate the strong convergence rate of the two classes of theta-Euler schemes for the case $\theta \in(1 / 2,1]$.

Theorem 3.4. Let Assumption 3.1 hold. Then for any $\Delta<\Delta^{*}$ and each integer $p \geqslant 2$,

$$
\mathbb{E}\left[\sup _{t \in[0, T]}|x(t)-z(t)|^{p}\right] \leqslant C \Delta^{p / 2}
$$

and

$$
\mathbb{E}\left[\sup _{k \Delta \in[0, T]}\left|x\left(t_{k}\right)-y_{k}\right|^{p}\right] \leqslant C \Delta^{p / 2}
$$

hold.

Before proving Theorem 3.4, we introduce some necessary lemmas. 
Lemma 3.5. For any $\Delta<\Delta^{*}$ and each integer $p \geqslant 2$, we have

$$
\mathbb{E}\left[\sup _{t \in[0, T]}|z(t)|^{p}\right] \leqslant C .
$$

Proof. For any $\mathrm{p} \geqslant 2$, by the integral form (3.17), we have

$$
\begin{aligned}
\mathbb{E}\left(\sup _{s \in[0, t]}|z(s)|^{p}\right) \leqslant & 4^{p-1} \mathbb{E}|z(0)|^{p}+4^{p-1} \mathbb{E}\left|\int_{0}^{t} f(y(\check{s}), y(\check{s}-\tau)) d s\right|^{p} \\
& +4^{p-1} \mathbb{E}\left(\sup _{s \in[0, t]}\left|\int_{0}^{s} g(y(\check{u}, y(\check{u}-\tau))) d w(u)\right|^{p}\right) .
\end{aligned}
$$

Resorting to (3.5) and Theorem 3.3, we can easily obtain

$$
\mathbb{E}\left|\int_{0}^{t} f(y(\check{s}), y(\check{s}-\tau)) d s\right|^{p} \leqslant C \mathbb{E} \int_{0}^{t}|f(y(\check{s}), y(\check{s}-\tau))|^{p} d s \leqslant C .
$$

Using the Burkholder-Davis-Gundy inequality, (3.5) and Theorem 3.3 gives

$$
\begin{aligned}
\mathbb{E}\left(\sup _{s \in[0, t]}\left|\int_{0}^{s} g(y(\check{u}, y(\check{u}-\tau))) d w(u)\right|^{p}\right) & \leqslant c_{p} \mathbb{E}\left(\int_{0}^{t}|g(y(\check{s}), y(\check{s}-\tau))|^{2} d s\right)^{p / 2} \\
& \leqslant C \int_{0}^{t} \mathbb{E}|g(y(\check{s}), y(\check{s}-\tau))|^{p} d s \leqslant C
\end{aligned}
$$

where

$$
c_{p}= \begin{cases}4, & p=2 \\ {\left[p^{p+1} / 2(p-1)^{p-1}\right]^{p / 2},} & p>2 .\end{cases}
$$

Therefore, combining (3.21)-(3.22) and (3.20) yields

$$
\mathbb{E} \sup _{s \in[0, T]}|z(s)|^{p} \leqslant C .
$$

Lemma 3.6. Assume all the conditions in Theorem 3.4 hold. Then for any $\mathrm{p} \geqslant 2, \mathrm{t} \in[0, \mathrm{~T}], \Delta<\Delta^{*}$,

$$
\begin{aligned}
& \mathbb{E}|x(\mathrm{t})-x(\check{\mathrm{t}})|^{p} \leqslant C \Delta^{\mathrm{p} / 2}, \\
& \mathbb{E}|z(\mathrm{t})-z(\check{\mathrm{t}})|^{p} \leqslant C \Delta^{\mathrm{p} / 2},
\end{aligned}
$$

and

$$
\mathbb{E}|z(\check{\mathrm{t}})-y(\check{\mathrm{t}})|^{\mathrm{p}} \leqslant \mathrm{C} \Delta^{\mathrm{p} / 2}
$$

hold.

Proof. By Assumption 3.1, Theorem 3.3, and the Burkholder-Davis-Gundy inequality, it is easy to show

$$
\begin{aligned}
& \mathbb{E}|x(t)-x(\check{\mathfrak{t}})|^{p}=C \mathbb{E}\left|\int_{\check{\mathfrak{t}}}^{t} f(x(s), x(s-\tau)) d s\right|^{p}+C \mathbb{E}\left|\int_{\check{t}}^{t} g(x(s), x(s-\tau)) d w(s)\right|^{p} \\
& \leqslant C \Delta^{p-1} \int_{\check{\mathfrak{t}}}^{\mathrm{t}} \mathbb{E}|\mathrm{f}(x(s), \chi(s-\tau))|^{\mathrm{p}} \mathrm{d} s+\left.\left.\mathrm{CE}\left|\int_{\check{\mathfrak{t}}}^{\mathrm{t}}\right| \mathrm{g}(\chi(s), \chi(s-\tau))\right|^{2} \mathrm{ds}\right|^{\mathrm{p} / 2} \leqslant \mathrm{C} \Delta^{\mathrm{p} / 2} .
\end{aligned}
$$

Similarly,

$$
\mathbb{E}|z(\mathrm{t})-z(\check{\mathrm{t}})|^{p}=\mathrm{C} \Delta^{p} \mathbb{E}|\mathrm{f}(\mathrm{y}(\check{\mathrm{t}}), y(\check{\mathrm{t}}-\tau))|^{p}+\mathrm{C} \mathbb{E}|\mathrm{g}(\mathrm{y}(\check{\mathrm{t}}), \mathrm{y}(\check{\mathrm{t}}-\tau))(w(\mathrm{t})-w(\check{\mathrm{t}}))|^{\mathrm{p}}
$$




$$
\leqslant C \Delta^{p} \mathbb{E}|f(y(\check{t}), y(\check{t}-\tau))|^{p}+C \Delta^{p / 2} \mathbb{E}|g(y(\check{t}), y(\check{\mathfrak{t}}-\tau))|^{p} \leqslant C \Delta^{p / 2} .
$$

Applying $z(\check{\mathrm{t}})-\mathrm{y}(\check{\mathrm{t}})=-\beta_{2} \Delta \mathrm{f}(\mathrm{y}(\check{\mathrm{t}}), \mathrm{y}(\check{\mathrm{t}}-\tau))+\beta_{0} \Delta \mathrm{f}(\mathrm{y}(\check{\mathrm{t}}), \mathrm{y}(\check{\mathrm{t}}-\tau))$ and Theorem 3.3 produces (3.23), where $\check{\mathrm{t}}=\mathrm{t}_{\mathrm{k}-1}$ for $\mathrm{t} \in\left[\mathrm{t}_{\mathrm{k}}, \mathrm{t}_{\mathrm{k}+1}\right), \mathrm{k}=1,2, \ldots$

We now give the proof of Theorem 3.4.

Proof of Theorem 3.4. Define $e(t)=x(t)-z(t)$. By (3.17) and (1.1), we have

$$
\begin{aligned}
e(t)= & e(0)+\int_{0}^{t}[f(x(s), x(s-\tau))-f(y(\check{s}), y(\check{s}-\tau))] d s \\
& +\int_{0}^{t}[g(x(s), x(s-\tau))-g(y(\check{s}), y(\check{s}-\tau))] d w(s) .
\end{aligned}
$$

Let $F(s)=f(x(s), x(s-\tau))-f(y(\check{s}), y(\check{s}-\tau))$ and $G(s)=g(x(s), x(s-\tau))-g(y(\check{s}), y(\check{s}-\tau))$. For any $p \geqslant 2$, we have

$$
|e(t)|^{p} \leqslant 3^{p-1}|e(0)|^{p}+3^{p-1}\left|\int_{0}^{t} F(s) d s\right|^{p}+3^{p-1}\left|\int_{0}^{t} G(s) d w(s)\right|^{p} .
$$

By Assumption 3.1, we can obtain that for any $\Delta<\Delta^{*}$

$$
\begin{aligned}
\mathbb{E}|\mathrm{F}(s)|^{p} \leqslant & a^{p} \mathbb{E}|x(s)-y(\check{s})|^{p}+\mathbb{E}\left(V_{1}(x(s-\tau), y(\check{s}-\tau))^{p}|x(s-\tau)-y(\check{s}-\tau)|^{p}\right) \\
\leqslant & 3^{p-1} a^{p}\left(\mathbb{E}|x(s)-z(s)|^{p}+\mathbb{E}|z(s)-z(\check{s})|^{p}+\mathbb{E}|z(\check{s})-y(\check{s})|^{p}\right) \\
& +\left(\mathbb{E} V_{1}(x(s-\tau), y(\check{s}-\tau))^{2 \mathfrak{p}} \mathbb{E}|x(s-\tau)-y(\check{s}-\tau)|^{2 p}\right)^{1 / 2} \\
\leqslant & C \mathbb{E}|e(s)|^{p}+C \sqrt{\mathbb{E}|e(s-\tau)|^{2 \mathfrak{p}}}+C \Delta^{p / 2}
\end{aligned}
$$

and

$$
\begin{aligned}
\mathbb{E}|G(s)|^{p} \leqslant & b^{p} \mathbb{E}|x(s)-y(\check{s})|^{p}+\mathbb{E}\left(V_{2}(x(s-\tau), y(\check{s}-\tau))^{p}|x(s-\tau)-y(\check{s}-\tau)|^{p}\right) \\
\leqslant & 3^{p-1} b^{p}\left(\mathbb{E}|x(s)-z(s)|^{p}+\mathbb{E}|z(s)-z(\check{s})|^{p}+\mathbb{E}|z(\check{s})-y(\check{s})|^{p}\right) \\
& +\left(\mathbb{E} V_{2}(x(s-\tau), y(\check{s}-\tau))^{2 \mathfrak{p}} \mathbb{E}|x(s-\tau)-y(\check{s}-\tau)|^{2 p}\right)^{1 / 2} \\
\leqslant & C \mathbb{E}|e(s)|^{p}+C \sqrt{\mathbb{E}|e(s-\tau)|^{2 p}}+C \Delta^{p / 2}
\end{aligned}
$$

where we also used Lemma 3.6 and Theorem 3.3. By the Hölder inequality, we have that for any $\Delta<\Delta^{*}$ and $r \in[0, T]$

$$
\mathbb{E}\left[\sup _{t \in[0, r]}\left|\int_{0}^{t} F(s) d s\right|^{p}\right] \leqslant C \mathbb{E} \int_{0}^{r}|F(s)|^{p} d s \leqslant C \int_{0}^{r} \mathbb{E}|e(s)|^{p} d s+C \int_{0}^{r} \sqrt{\mathbb{E}|e(s-\tau)|^{2 p}} d s+C \Delta^{p / 2} .
$$

Resorting to the Burkholder-Davis-Gundy inequality and the Hölder inequality, we have that for any $\Delta<\Delta^{*}$

$$
\begin{aligned}
\mathbb{E}\left[\sup _{t \in[0, r]}\left|\int_{0}^{t} G(s) d w(s)\right|^{p}\right] & \leqslant c_{p} \mathbb{E}\left(\int_{0}^{r}|G(s)|^{2} d s\right)^{p / 2} \\
& \leqslant c_{p} T^{p / 2-1} \int_{0}^{r} \mathbb{E}|G(s)|^{p} d s \\
& \leqslant C \int_{0}^{r} \mathbb{E}|e(s)|^{p} d s+C \int_{0}^{r} \sqrt{\mathbb{E}|e(s-\tau)|^{2 p}} d s+C \Delta^{p / 2},
\end{aligned}
$$


where

$$
c_{p}= \begin{cases}4, & p=2, \\ {\left[(p)^{p+1} / 2(p-1)^{p-1}\right]^{p / 2},} & p>4 .\end{cases}
$$

Combining (3.25), (3.26), and (3.24) produces that for any $\Delta<\Delta^{*}$

$$
\mathbb{E}\left[\sup _{t \in[0, r]}|e(t)|^{p}\right] \leqslant C \int_{0}^{r} \mathbb{E} \sup _{u \in[0, s]}|e(u)|^{p} d s+C \int_{0}^{r} \sqrt{\mathbb{E}|e(s-\tau)|^{2 p}} d s+C \Delta^{p / 2} .
$$

Hence, the continuous Gronwall inequality gives that for any $\Delta<\Delta^{*}$,

$$
\mathbb{E}\left[\sup _{t \in[0, r]}|e(t)|^{p}\right] \leqslant C \int_{0}^{r} \sqrt{\mathbb{E}|e(s-\tau)|^{2 p}} d s+C \Delta^{p / 2} .
$$

For any $p \geqslant 2$, we define

$$
p_{i}=([T / \tau]+2-i) p 2^{[T / \tau]+1-i}, i=1,2, \ldots,[T / \tau]+1,
$$

where $[a]$ denotes the integer part of the real number a. It is easy to see that

$$
2 p_{i+1}<p_{i} \text { and } p_{[T / \tau]+1}=p, i=1,2, \ldots,[T / \tau] .
$$

Noting that $\mathbb{E}|e(s)|^{2 p} \leqslant C \Delta^{2 p}$ for $s \in[-\tau, 0]$ and any $p>0$ since $z(s)=\chi(s)-\theta \Delta f(x(s), \chi(s-\tau))$ for $s \in[-\tau, 0]$, we have from (3.27) that

$$
\mathbb{E}\left[\sup _{t \in[0, \tau]}|e(t)|^{p_{1}}\right] \leqslant C \int_{0}^{\tau} \sqrt{\mathbb{E}|e(s-\tau)|^{2 p_{1}}} d s+C \Delta^{p_{1} / 2} \leqslant C \Delta^{p_{1} / 2},
$$

which together with (3.28), (3.29), and the Hölder inequality gives that for any $\Delta<\Delta^{*}$,

$$
\begin{aligned}
\mathbb{E}\left[\sup _{t \in[0,2 \tau]}|e(t)|^{p_{2}}\right] & \leqslant C \int_{0}^{2 \tau} \sqrt{\mathbb{E}|e(s-\tau)|^{2 p_{2}}} d s+C \Delta^{p_{2} / 2} \\
& \leqslant C \int_{0}^{2 \tau}\left(\mathbb{E}|e(s-\tau)|^{p_{1}}\right)^{\frac{p_{2}}{p_{1}}} \mathrm{~d} s+C \Delta^{p_{2} / 2} \leqslant C \Delta^{p_{2} / 2} .
\end{aligned}
$$

The desired assertion (3.18) then follows by repeating the previous procedures. Combining (3.18), Lemma 3.5, and $z_{k}=y_{k}-\beta_{2} f\left(y_{k}, y_{k-m}\right) \Delta-\beta_{0} f\left(y_{k-1}, y_{k-1-m}\right) \Delta$ gives (3.19).

Remark 3.7. Buckwar and Winkler [8] proved that under the global Lipschitz condition, the LTSM scheme is mean square convergent, that is, $\sup _{k \Delta \in[0, T]} \mathbb{E}\left|y_{k}-x\left(t_{k}\right)\right|^{2} \leqslant C \Delta$. Hence, Theorem 3.4 improves the convergence results in [8]. We obtain the optimal strong convergence rate of the two-step schemes for SDDEs with highly nonlinear delay variable. These convergence results also hold for SODEs with global Lipschitz coefficients.

After the convergence rate of the two-step Maruyama schemes, we now establish their stability analysis.

\section{Exponential mean square stability}

For the purpose of stability, without loss of generality, we assume $f(0)=0$ and $g(0)=0$. This shows that SDE (1.1) admits a trivial solution. We also assume that $f$ and $g$ satisfy the following local Lipschitz condition. 
Assumption 4.1. $f$ and $g$ satisfy the local Lipschitz condition, that is, for each $j>0$ there exists a positive constant $K_{j}$ such that for any $x, y, \bar{x}, \bar{y} \in \mathbb{R}^{n}$ with $|x| \vee|y| \vee|\bar{y}| \vee|\bar{y}| \leqslant j$,

$$
|f(x, y)-f(\bar{x}, \bar{y})| \vee|g(x, y)-g(\bar{x}, \bar{y})| \leqslant K_{j}(|x-\bar{x}|+|y-\bar{y}|) .
$$

The local Lipschitz condition together with the linear growth condition or a class of monotone condition (for example, condition (4.1) below) may guarantee that Eq. (1.1) admits a global solution (see [21]). Let us firstly present the following theorem which gives the stability criterion of the SDDE (1.1) (also see [21]).

Theorem 4.2. Let Assumption 4.1 hold. If there exist nonnegative constants $\mu>\sigma \geqslant 0$ such that for all $x, y \in \mathbb{R}^{n}$,

$$
2 x^{\top} f(x, y)+|g(x, y)|^{2} \leqslant-\mu|x|^{2}+\sigma|y|^{2},
$$

then the solution to (1.1) has the property

$$
\mathbb{E}|x(t)|^{2} \leqslant C(\varphi) e^{-\gamma t}
$$

where $\mathrm{C}(\varphi)$ is a positive constant dependent on the initial data $\varphi$, and $\gamma$ is the unique root of equation

$$
\gamma+\sigma e^{\gamma \tau}-\mu=0
$$

This section aims to examine whether the two classes of two-step Maruyama schemes that can replicate the exponential mean square stability of the exact solution to nonlinear SDDEs.

Theorem 4.3. Let all the conditions in Theorem 4.2 hold, and $\mathrm{f}$ satisfies the linear growth condition

$$
|f(x, y)|^{2} \leqslant K\left(|x|^{2}+|y|^{2}\right) .
$$

Then there exists a positive stepsize $\Delta^{* *}$ such that for any $\Delta<\Delta^{* *}$, STSM scheme (2.3) satisfies

$$
\mathbb{E}\left|z_{k}\right|^{2} \leqslant \mathrm{C}\left(x_{0}\right) e^{-\gamma_{\Delta} k \Delta},
$$

where $\gamma_{\Delta} \in(0,(1 / \tau) \log (\mu / \sigma))$ and satisfies

$$
\lim _{\Delta \rightarrow 0} \gamma_{\Delta}=\gamma
$$

Proof. By STSM scheme (2.3), we have

$$
\begin{aligned}
\left|z_{k+1}\right|^{2} \leqslant & \left|z_{k}\right|^{2}+2 z_{k}^{\top} f\left(y_{k}, y_{k-m}\right) \Delta+\left|g\left(y_{k}, y_{k-m}\right) \Delta w_{k}\right|^{2} \\
& +\left|f\left(y_{k}, y_{k-m}\right) \Delta\right|^{2}+2\left\langle z_{k}+f\left(y_{k}, y_{k-m}\right) \Delta, g\left(y_{k}, y_{k-m}\right) \Delta w_{k}\right\rangle .
\end{aligned}
$$

Note that $z_{k}=y_{k}-\beta_{2} f\left(y_{k}, y_{k-m}\right) \Delta+\beta_{0} f\left(y_{k-1}, y_{k-1-m}\right) \Delta$. Substituting this into (4.4) yields

$$
\begin{aligned}
\left|z_{k+1}\right|^{2} \leqslant & \left|z_{k}\right|^{2}+2 y_{k}^{\top} f\left(y_{k}, y_{k-m}\right) \Delta-2 \beta_{0} f\left(y_{k-1}, y_{k-1-m}\right)^{\top} f\left(y_{k}, y_{k-m}\right) \Delta^{2}+\left|g\left(y_{k}, y_{k-m}\right) \Delta w_{k}\right|^{2} \\
& +\left(1-2 \beta_{2}\right)\left|f\left(y_{k}, y_{k-m}\right) \Delta\right|^{2}+2\left\langle z_{k}+f\left(y_{k}, y_{k-m}\right) \Delta, g\left(y_{k}, y_{k-m}\right) \Delta w_{k}\right\rangle \\
\leqslant & \left|z_{k}\right|^{2}-\mu \Delta\left|y_{k}\right|^{2}+\sigma \Delta\left|y_{k-m}\right|^{2}+\left|\beta_{0}\right| K\left(\left|y_{k-1}\right|^{2}+\left|y_{k}\right|^{2}+\left|y_{k-1-m}\right|^{2}+\left|y_{k-m}\right|^{2}\right) \Delta^{2} \\
& +\left|1-2 \beta_{2}\right| K\left(\left|y_{k}\right|^{2}+\left|y_{k-m}\right|^{2}\right) \Delta^{2}+m_{k},
\end{aligned}
$$

where $m_{k}=2\left\langle z_{k}+f\left(y_{k}, y_{k-m}\right) \Delta, g\left(y_{k}, y_{k-m}\right) \Delta w_{k}\right\rangle+\left|g\left(y_{k}, y_{k-m}\right)\right|^{2}\left(\left|\Delta w_{k}\right|^{2}-\Delta\right)$. Note that $\mathbb{E} m_{k}=0$. Taking expectations on the both sides of inequality (4.5) produces

$$
\begin{aligned}
\mathbb{E}\left|z_{k+1}\right|^{2} \leqslant & \mathbb{E}\left|z_{k}\right|^{2}+\left[-\mu+\left(\left|\beta_{0}\right|+\left|1-2 \beta_{2}\right|\right) K \Delta\right] \Delta \mathbb{E}\left|y_{k}\right|^{2}+K\left|\beta_{0}\right| \Delta^{2} \mathbb{E}\left|y_{k-1}\right|^{2} \\
& +\left[\sigma+\left(\left|\beta_{0}\right|+\left|1-2 \beta_{2}\right|\right) K \Delta\right] \Delta \mathbb{E}\left|y_{k-m}\right|^{2}+K\left|\beta_{0}\right| \Delta^{2} \mathbb{E}\left|y_{k-1-m}\right|^{2} .
\end{aligned}
$$

Define $\bar{\mu}_{\Delta}=\mu-\left(\left|\beta_{0}\right|+\left|1-2 \beta_{2}\right|\right) K \Delta, \bar{\sigma}_{\Delta}=\sigma+\left(\left|\beta_{0}\right|+\left|1-2 \beta_{2}\right|\right) K \Delta$. For any positive number $\mathrm{O}>1$, we 
have

$$
\begin{aligned}
\mathrm{O}^{(\mathrm{k}+1) \Delta} \mathbb{E}\left|z_{\mathrm{k}+1}\right|^{2}-\mathrm{O}^{\mathrm{k} \Delta} \mathbb{E}\left|z_{\mathrm{k}}\right|^{2} \leqslant & \left(1-\mathrm{O}^{-\Delta}\right) \mathrm{O}^{(\mathrm{k}+1) \Delta} \mathbb{E}\left|z_{\mathrm{k}}\right|^{2}-\bar{\mu}_{\Delta} \Delta \mathrm{O}^{(\mathrm{k}+1) \Delta} \mathbb{E}\left|\mathrm{y}_{\mathrm{k}}\right|^{2} \\
& +\overline{\mathrm{o}}_{\Delta} \Delta \mathrm{O}^{(\mathrm{k}+1) \Delta} \mathbb{E}\left|\mathrm{y}_{\mathrm{k}-\mathrm{m}}\right|^{2}+\mathrm{K}\left|\beta_{0}\right| \Delta^{2} \mathrm{O}^{(\mathrm{k}+1) \Delta}\left(\mathbb{E}\left|\mathrm{y}_{\mathrm{k}-1}\right|^{2}+\mathbb{E}\left|\mathrm{y}_{\mathrm{k}-1-\mathrm{m}}\right|^{2}\right),
\end{aligned}
$$

which implies

$$
\begin{aligned}
\mathrm{O}^{(\mathrm{k}+1) \Delta} \mathbb{E}\left|z_{k+1}\right|^{2} \leqslant & \mathbb{E}\left|z_{0}\right|^{2}+\left(1-\mathrm{O}^{-\Delta}\right) \sum_{j=0}^{\mathrm{k}} \mathrm{O}^{(j+1) \Delta} \mathbb{E}\left|z_{j}\right|^{2}+\bar{\sigma}_{\Delta} \Delta \sum_{j=0}^{k} \mathrm{O}^{(j+1) \Delta} \mathbb{E}\left|y_{j-m}\right|^{2} \\
& -\bar{\mu}_{\Delta} \Delta \sum_{j=0}^{k} \mathrm{O}^{(j+1) \Delta} \mathbb{E}\left|y_{j}\right|^{2}+\mathrm{K}\left|\beta_{0}\right| \Delta^{2} \sum_{j=0}^{k} \mathrm{O}^{(j+1) \Delta} \mathbb{E}\left|y_{j-1}\right|^{2} \\
& +\mathrm{K}\left|\beta_{0}\right| \Delta^{2} \sum_{j=0}^{k} \mathrm{O}^{(j+1) \Delta} \mathbb{E}\left|y_{j-1-m}\right|^{2}
\end{aligned}
$$

Noting that

$$
\begin{aligned}
\left|z_{j}\right|^{2}= & \left|y_{j}\right|^{2}+2 \Delta y_{j}^{\top}\left[\beta_{2} f\left(y_{j}, y_{j-m}\right)+\beta_{0} f\left(y_{j-1}, y_{j-1-m}\right)\right] \\
& +\left|\beta_{2} f\left(y_{j}, y_{j-m}\right)+\beta_{0} f\left(y_{j-1}, y_{j-1-m}\right)\right|^{2} \Delta^{2} \\
\leqslant & \left|y_{j}\right|^{2}+\Delta\left|y_{j}\right|^{2}+2\left|\beta_{2} f\left(y_{j}, y_{j-m}\right)+\beta_{0} f\left(y_{j-1}, y_{j-1-m}\right)\right|^{2} \Delta^{2} \\
\leqslant & \left(1+\Delta+4\left|\beta_{2}\right| K \Delta^{2}\right)\left|y_{j}\right|^{2}+4\left|\beta_{2}\right| K \Delta^{2}\left|y_{j-m}\right|^{2}+4\left|\beta_{0}\right| K \Delta^{2}\left(\left|y_{j-1}\right|^{2}+\left|y_{j-1-m}\right|^{2}\right),
\end{aligned}
$$

then we have from (4.6) that

$$
\begin{aligned}
\mathrm{O}^{(\mathrm{k}+1) \Delta} \mathbb{E}\left|z_{k+1}\right|^{2} \leqslant & \mathbb{E}\left|z_{0}\right|^{2}+\left(1+\Delta+4\left|\beta_{2}\right| \mathrm{K} \Delta^{2}\right)\left(1-\mathrm{O}^{-\Delta}\right) \sum_{j=0}^{\mathrm{k}} \mathrm{O}^{(j+1) \Delta} \mathbb{E}\left|\mathrm{y}_{j}\right|^{2} \\
& +\left[\bar{\sigma}_{\Delta}+4\left|\beta_{2}\right| \mathrm{K} \Delta\right] \Delta \sum_{j=0}^{\mathrm{k}} \mathrm{O}^{(j+1) \Delta} \mathbb{E}\left|\mathrm{y}_{j-m}\right|^{2}-\bar{\mu}_{\Delta} \Delta \sum_{j=0}^{\mathrm{k}} \mathrm{O}^{(j+1) \Delta} \mathbb{E}\left|\mathrm{y}_{j}\right|^{2} \\
& +5 \mathrm{~K}\left|\beta_{0}\right| \Delta^{2} \sum_{j=0}^{k} \mathrm{O}^{(j+1) \Delta} \mathbb{E}\left|y_{j-1}\right|^{2}+5 \mathrm{~K}\left|\beta_{0}\right| \Delta^{2} \sum_{j=0}^{k} \mathrm{O}^{(j+1) \Delta} \mathbb{E}\left|y_{j-1-m}\right|^{2}
\end{aligned}
$$

Note that

$$
\begin{aligned}
& \sum_{j=0}^{k} \mathrm{O}^{(j+1) \Delta}\left|y_{j-m}\right|^{2}=\mathrm{O}^{\tau} \sum_{j=-m}^{-1} \mathrm{O}^{(j+1) \Delta}\left|y_{j}\right|^{2}+\mathrm{O}^{\tau} \sum_{j=0}^{k-m} \mathrm{O}^{(j+1) \Delta}\left|y_{j}\right|^{2} \\
& \leqslant \mathrm{O}^{\tau} \sum_{j=-m}^{-1}\left|y_{j}\right|^{2}+\mathrm{O}^{\tau} \sum_{j=0}^{k} \mathrm{O}^{(j+1) \Delta}\left|y_{j}\right|^{2}, \\
& \sum_{j=0}^{k} \mathrm{O}^{(j+1) \Delta} \mathbb{E}\left|y_{j-1}\right|^{2}=\mathrm{O}^{\Delta}\left|y_{-1}\right|^{2}+\mathrm{O}^{\Delta} \sum_{j=0}^{k-1} \mathrm{O}^{\left.(j+1) \Delta_{\mid y_{j}}\right|^{2}} \leqslant \mathrm{O}^{\Delta}\left|y_{-1}\right|^{2}+\mathrm{O}^{\Delta} \sum_{j=0}^{k} \mathrm{O}^{(j+1) \Delta}\left|y_{j}\right|^{2},
\end{aligned}
$$

and

$$
\sum_{j=0}^{k} \mathrm{O}^{(j+1) \Delta}\left|y_{j-1-m}\right|^{2}=\sum_{j=-m-1}^{k-m-1} \mathrm{O}^{(j+m+2) \Delta}\left|y_{j}\right|^{2} \leqslant \mathrm{O}^{\tau+\Delta} \sum_{j=-m}^{-1}\left|y_{j}\right|^{2}+\mathrm{O}^{\tau+\Delta} \sum_{j=0}^{k} \mathrm{O}^{(j+1) \Delta}\left|y_{j}\right|^{2} .
$$


Let

$$
\mathrm{h}(\mathrm{O})=\left(1+\Delta+4\left|\beta_{2}\right| \mathrm{K} \Delta^{2}\right) \frac{1-\mathrm{O}^{-\Delta}}{\Delta}-\bar{\mu}_{\Delta}+\left(\bar{\sigma}_{\Delta}+4\left|\beta_{2}\right| \mathrm{K} \Delta\right) \mathrm{O}^{\tau}+5\left|\beta_{0}\right| \mathrm{K} \Delta \mathrm{O}^{\Delta}+5\left|\beta_{0}\right| \mathrm{K} \Delta \mathrm{O}^{\Delta+\tau} .
$$

Hence, we can obtain from (4.7)-(4.10) that

$$
\mathrm{O}^{(\mathrm{k}+1) \Delta} \mathbb{E}\left|z_{\mathrm{k}+1}\right|^{2} \leqslant \mathrm{C}(\varphi)+\mathrm{h}(\mathrm{O}) \Delta \sum_{j=0}^{\mathrm{k}} \mathrm{O}^{(j+1) \Delta} \mathbb{E}\left|\mathrm{y}_{j}\right|^{2},
$$

where $C(\varphi)$ is a constant depending on the initial data $\varphi$. Let $\Delta^{* *}=(\mu-\sigma) /\left[\left(12\left|\beta_{2}\right|+2+8\left|\beta_{2}\right|\right) \mathrm{K}\right]$. Then for $\Delta<\Delta^{* *}$,

$$
h(1)=-\mu+2\left(\left|\beta_{0}\right|+\left|1-2 \beta_{2}\right|\right) K \Delta+\sigma+4\left|\beta_{2}\right| K \Delta+10\left|\beta_{0}\right| K \Delta \leqslant-\mu+\sigma+\left(12\left|\beta_{0}\right|+2+8\left|\beta_{2}\right|\right) K \Delta<0 .
$$

Let $\overline{\mathrm{O}}=(\mu / \sigma)^{1 / \tau}$, then $\mathrm{h}(\overline{\mathrm{O}})>\mu-\sigma \overline{\mathrm{O}}^{\tau}=0$. Moreover, $\mathrm{h}^{\prime}(\mathrm{O})>0$ for all $\mathrm{O} \in(1, \overline{\mathrm{O}})$. Therefore, for any $\Delta<\Delta^{* *}$, there exists a positive constant $\mathrm{O}^{*}(\Delta) \in\left(1,(\mu / \sigma)^{1 / \tau}\right)$ such that $\mathrm{h}\left(\mathrm{O}^{*}(\Delta)\right)=0$ and $\mathrm{h}(\mathrm{O})<0$ for $\mathrm{O}<\mathrm{O}^{*}(\Delta)$. Let $\gamma_{\Delta}=\log \mathrm{O}^{*}(\Delta)$. By (4.11), we have that for any $\Delta<\Delta^{* *}$,

$$
e^{\gamma \Delta k \Delta} \mathbb{E}\left|z_{k}\right|^{2} \leqslant C(\varphi)
$$

It is easy to see from $h\left(\mathrm{O}^{*}(\Delta)\right)=0$ that limitation (4.3) holds.

Based on Theorem 4.3, we now investigate the exponential mean stability of the SLTSM scheme.

Theorem 4.4. Let all the conditions in Theorem 4.2 hold. If $\mathrm{f}$ satisfies the linear growth condition (4.2), then for any $\Delta<\Delta^{* *}$, SLTE scheme (2.2) has the property

$$
\mathbb{E}\left|y_{k}\right|^{2} \leqslant C e^{-\left(\gamma_{\Delta}-\epsilon\right) k \Delta},
$$

where $\mathrm{C}$ is a positive constant, $\Delta^{* *}$ is defined in Theorem $4.3, \gamma_{\Delta}$ is defined in Theorem 4.3 and satisfies the limit (4.3), and $\epsilon \in\left(0, \gamma_{\Delta}\right)$ is a sufficiently small constant.

Proof. By Theorem 4.3, we have from (4.11) that for any fixed $\Delta<\bar{\Delta}$ and any sufficiently small $\varepsilon$, $\mathrm{h}\left(e^{\left(\gamma_{\Delta}-\varepsilon\right)}\right)<0$ and

$$
e^{\left(\gamma_{\Delta}-\varepsilon\right) k \Delta} \mathbb{E}\left|z_{k}\right|^{2} \leqslant C(\varphi)+h\left(e^{\left(\gamma_{\Delta}-\varepsilon\right)}\right) \Delta \sum_{j=0}^{k} e^{\left(\gamma_{\Delta}-\varepsilon\right)(j+1) \Delta} \mathbb{E}\left|y_{j}\right|^{2}, \forall k>0 .
$$

Hence, there exists a constant $C$ such that $\sum_{j=0}^{k} e^{\left(\gamma_{\Delta}-\varepsilon\right)(j+1) \Delta} \mathbb{E}\left|y_{j}\right|^{2} \leqslant C$ for any $k>0$, which implies (4.12).

Remark 4.5. Cao and Zhang [9] obtained the exponential mean square stability of LTSM scheme for linear SDDE. Theorem 4.4 shows that the LSTM scheme can not only share the exponential mean square stability of the exact solution, but also preserve the bound of Lyapunov exponent for sufficient small stepsize. The existed works $[5,27]$ are devoted to the asymptotic mean square stability of the linear two-step Maruyama schemes for the linear SODEs. For nonlinear SODEs (the delay vanishes), Theorem 4.4 further takes the Lyapunov exponent of mean square stability into consideration. 


\section{Concluding remark}

In this paper, the split two-step Maruyama and linear two-step schemes have been considered for nonlinear SDDEs. It is shown that the two classes of two-step Maruyama schemes converge strongly to the exact solution of the SDDEs with highly nonlinear delay variables and the convergence rate is 0.5 . Exponential mean square stability and exponential decay rate of the two classes of two-step Maruyama schemes are also investigated. It is proved that they can not only inherit the exponential mean square stability of the exact solution, but also preserve the bound of the second moment Lyapunov exponent for sufficient small stepsize, which may measure the decay rate of the numerical solutions. When the delay vanishes, these results also hold for nonlinear SODEs.

Note that these convergence results are based on the global Lipschitz condition in the present states of the coefficients. When $\beta_{0}=0$ and $\beta_{2} \in[0,1]$, STSM and LTSM schemes are equivalent respectively with the split step theta-Euler (SSTE) and stochastic linear theta-Euler (SLTE) schemes studied in [34]. Moreover, the strong convergence rate and exponential mean square stability of the theta-Euler schemes were obtained for SDDEs with non-global Lipschitz condition in not only the delay states, but also the present states. Hence, it is interesting to question whether the multi-step scheme can be applied to the SDDEs or SODEs with non-global Lipschitz present states. And we hope to be able to discuss this question elsewhere.

\section{Acknowledgment}

The authors would like to thank the editor and the anonymous reviewers for their very useful comments and suggestions. The work was partially supported by the Natural Science Foundation of China under Grants (11422110, 61703378, 61374085) and the Fundamental Research Funds for the Central Universities, South-Central University for Nationalities under Grant CZW15113.

\section{References}

[1] Y. Ait-Sahalia, Testing continuous-time models of the spot interest rate, Rev. Financial Stud., 9 (1996), 385-426. 1

[2] J.-H. Bao, C.-G. Yuan, Convergence rate of EM scheme for SDDEs, Proc. Amer. Math. Soc., 141 (2013), 3231-3243. 3

[3] E. Beretta, V. Kolmanovskii, L. Shaikhet, Stability of epidemic model with time delays influenced by stochastic perturbations, Delay systems, Lille, (1996), Math. Comput. Simulation, 45 (1998), 269-277. 1

[4] A. Bryden, D. J. Higham, On the boundedness of asymptotic stability regions for the stochastic theta method, BIT, 43 (2003), 1-6. 1

[5] E. Buckwar, R. Horváth-Bokor, R. Winkler, Asymptotic mean-square stability of two-step methods for stochastic ordinary differential equations, BIT, 46 (2006), 261-282. 1, 4.5

[6] E. Buckwar, R. Winkler, On two-step schemes for SDEs with small noise, PAMM, 4 (2004), 15-18. 1

[7] E. Buckwar, R. Winkler, Multistep methods for SDEs and their application to problems with small noise, SIAM J. Numer. Anal., 44 (2006), 779-803. 1

[8] E. Buckwar, R. Winkler, Multi-step Maruyama methods for stochastic delay differential equations, Stoch. Anal. Appl., 25 (2007), 933-959. 1, 2, 2, 3.7

[9] W.-R. Cao, Z.-Q. Zhang, On exponential mean-square stability of two-step Maruyama methods for stochastic delay differential equations, J. Comput. Appl. Math., 245 (2013), 182-193. 1, 4.5

[10] C. W. Eurich, J. G. Milton, Noise-induced transitions in human postural sway, Phys. Rev. E, 54 (1996), 6681-6684. 1

[11] D. J. Higham, Mean-square and asymptotic stability of the stochastic theta method, SIAM J. Numer. Anal., 38 (2000), 753-769. 1

[12] D. J. Higham, X.-R. Mao, A. M. Stuart, Strong convergence of Euler-type methods for nonlinear stochastic differential equations, SIAM J. Numer. Anal., 40 (2002), 1041-1063. 2

[13] D. J. Higham, X.-R. Mao, A. M. Stuart, Exponential mean-square stability of numerical solutions to stochastic differential equations, LMS J. Comput. Math., 6 (2003), 297-313. 1

[14] D. G. Hobson, L. C. G. Rogers, Complete models with stochastic volatility, Math. Finance, 8 (1998), 27-48. 1 
[15] C.-M. Huang, Exponential mean square stability of numerical methods for systems of stochastic differential equations, J. Comput. Appl. Math., 236 (2012), 4016-4026. 1

[16] C.-M. Huang, Mean square stability and dissipativity of two classes of theta methods for systems of stochastic delay differential equations, J. Comput. Appl. Math., 259 (2014), 77-86.

[17] M. Hutzenthaler, A. Jentzen, Numerical approximations of stochastic differential equations with non-globally Lipschitz continuous coefficients, Mem. Amer. Math. Soc., 236 (2015), 102 pages. 1

[18] P. E. Kloeden, E. Platen, Numerical solution of stochastic differential equations, Applications of Mathematics (New York), Springer-Verlag, Berlin, (1992). 1

[19] M.-Z. Liu, W.-R. Cao, Z.-C. Fan, Convergence and stability of the semi-implicit Euler method for a linear stochastic differential delay equation, J. Comput. Appl. Math., 170 (2004), 255-268. 1

[20] A. Longtin, J. G. Milton, J. E. Bos, M. C. Mackey, Noise and critical behavior of the pupil light reflex at oscillation onset, Phys. Rev. A, 41 (1990), 6992-7005. 1

[21] X.-R. Mao, Stochastic differential equations and their applications, Horwood Publishing Series in Mathematics \& Applications, Horwood Publishing Limited, Chichester, (1997). 4

[22] G. N. Milstein, Numerical integration of stochastic differential equations, Translated and revised from the 1988 Russian original, Mathematics and its Applications, Kluwer Academic Publishers Group, Dordrecht, (1995). 1

[23] G. N. Milstein, M. V. Tretyakov, Stochastic numerics for mathematical physics, Scientific Computation, SpringerVerlag, Berlin, (2004). 1

[24] Y. Saito, T. Mitsui, Stability analysis of numerical schemes for stochastic differential equations, SIAM J. Numer. Anal., 33 (1996), 2254-2267. 1

[25] T. Sickenberger, Mean-square convergence of stochastic multi-step methods with variable step-size, J. Comput. Appl. Math., 212 (2008), 300-319. 1

[26] P. K. Tapaswi, A. Mukhopadhyay, Effects of environmental fluctuation on plankton allelopathy, J. Math. Biol., 39 (1999), 39-58. 1

[27] A. Tocino, M. J. Senosiain, Asymptotic mean-square stability of two-step Maruyama schemes for stochastic differential equations, J. Comput. Appl. Math., 260 (2014), 337-348. 1, 4.5

[28] X.-J. Wang, S.-Q. Gan, The improved split-step backward Euler method for stochastic differential delay equations, Int. J. Comput. Math., 88 (2011), 2359-2378. 1

[29] X.-J. Wang, S.-Q. Gan, D.-S. Wang, A family of fully implicit Milstein methods for stiff stochastic differential equations with multiplicative noise, BIT, 52 (2012), 741-772. 1

[30] F.-K. Wu, X.-R. Mao, K. Chen, The Cox-Ingersoll-Ross model with delay and strong convergence of its Euler-Maruyama approximate solutions, Appl. Numer. Math., 59 (2009), 2641-2658. 1

[31] X.-F. Zong, F.-K. Wu, Choice of $\theta$ and mean-square exponential stability in the stochastic theta method of stochastic differential equations, J. Comput. Appl. Math., 255 (2014), 837-847. 1

[32] X.-F. Zong, F.-K. Wu, C.-M. Huang, Convergence and stability of the semi-tamed Euler scheme for stochastic differential equations with non-Lipschitz continuous coefficients, Appl. Math. Comput., 228 (2014), 240-250.

[33] X.-F. Zong, F.-K. Wu, C.-M. Huang, Preserving exponential mean square stability and decay rates in two classes of theta approximations of stochastic differential equations, J. Difference Equ. Appl., 20 (2014), 1091-1111. 1, 2

[34] X.-F. Zong, F.-K. Wu, C.-M. Huang, Theta-Euler schemes for SDEs with non-global Lipschitz continuous coeffcients, Submitted. 5 